\title{
Ground-based direct imaging of extra-solar planets supported by AO
}

\author{
Elena Masciadri ${ }^{1}$, Kerstin Geissler ${ }^{1}$, Stephan Kellner ${ }^{1}$, \\ Wolfgang Brandner ${ }^{1}$, Thomas Henning ${ }^{1}$, Reinhard Mundt ${ }^{1}$, \\ Laird Close ${ }^{2}$, Beth Biller ${ }^{2}$, and Alejandro Raga ${ }^{3}$ \\ ${ }^{1}$ Max-Planck Institut für Astronomie, Konigstuhl 17 - D69117, Heidelberg, Germany \\ email: masciadri@mpia.de \\ ${ }^{2}$ Steward Observatory, Tucson, Arizona \\ ${ }^{3}$ Ciencias Nucleares, UNAM, Mexico D.F., Mexico
}

\begin{abstract}
The detection of extra-solar planets made by direct imaging is an extremely challenging goal for astronomers due to the possibility to access the physical properties of planets and not only their existence. Using $8 \mathrm{~m}$ class telescopes joint with dedicated techniques (such as Simultaneous Differential Imaging aiming to suppress the speckle noise) it is possible, at present, to attain detection limit of 9-11 mag at 0.5 arcsec i.e. to access $3-10 \mathrm{M}_{J}$ planets orbiting around young (100-200 Myr) nearby and late type stars. Searches for extra-solar planets carried out with the present technology are quite fundamental and critical not only for discovery of planets but also because it permits us to put constraints on theories of planets formation and migration. Besides, our understandings of the performances of sophisticated techniques such as the SDI is fundamental to plan new observational strategies, new generation instruments and telescopes. Speckles noise is, indeed, the main source of noise for observations in the NIR and visible and our ability in suppressing it is not so easily scaled at different parameters space. In this contribution I will present the main results that we obtained in on-going searches for planets carried out with $\mathrm{NACO}$ and NACO/SDI in the last years. A particular attention will be dedicated in comparing different observational strategies and in the employment of image processing techniques for recognizing, in an automatic way, planet features in deep images obtained with ground-based telescopes and AO facilities.
\end{abstract}

Keywords. planetary systems, image processing.

\section{Introduction}

Adaptive optics (AO) imaging techniques at 8-10 meter telescopes can detect young planets if they are sufficiently distant from their parent star. Since it is reasonable to assume a coevality between the parent star and the planet, we can retrieve from atmospherical models (Burrows et al. 1997, Baraffe et al. 2003) that planets having a mass in the range $3-10 \mathrm{M}_{J}$ and orbiting around young late-type stars (10-200 Myr) have a typical brightness contrast with respect to the parent star of the order of $10^{2}-10^{6}$, corresponding to a magnitude difference of $\Delta M$ of $5-15$ mag. This means that a planet can be detected at a few tens of AUs from parent stars having distances of $\sim 50 \mathrm{pc}$.

According to recent statistical results (e.g. Marcy et al. 2003), most of known exoplanets orbit at distances smaller than $1 \mathrm{AU}$ and the semi-major axes of the exo-planets found so far are not larger than $\sim 6$ AU. Since these results are heavily biased towards short separations the derived major-axis distribution is certainly not representative. Not much is known at present about potential planets that could exist at distances larger than about 6 AU. Besides, there are models (for example the gravitational scattering model) that claim that in multi-planetary systems, due to gravitational interactions between the 
planets, it can happen that the lightest objects are ejected on hyperbolic trajectories and some planets can move on stable orbits having a distance of a few tens AU from the parent star. Such models were proposed by Weidenschilling \& Marzari (1996) and Rasio \& Ford (1996) and studied more recently by Papaloizou \& Terquem (2001).

There are thus elements that indicate that such planets might exist at large distances from the central star. For this reason we planned and performed an extended survey aiming to search for massive exo-planets orbiting around nearby and young stars using one of the best available instruments at the present time for high-contrast AO imaging observations: NACO at the Very Large Telescope (VLT). Once the survey completed (Masciadri et al. 2005), we decided a follow-up of our project employing the SDI/NACO instrument. As explained in Section 3, such an instrument permits to improve the contrast reachable at sub arcsecond distances from the parent star. I report here the principal results obtained and I discuss the performances of the different observational strategies. Finally, I deal about a method that a few of us (Masciadri \& Raga, 2004) developed for recognizing in an automatic way, planets features in deep images.

\section{Deep direct imaging survey}

We refer to Masciadri et al. (2005) for detailed informations on the target selection, observational strategy employed in the survey and data processing. Here we briefly summarize the principal informations. Around 30 targets (nearby stars) were observed during 3 runs. The targets are placed at a distance $d \leqslant 77 \mathrm{pc}$ (with $70 \%$ of them in the first $32 \mathrm{pc}$ ), they have an age of 1-200 Myr and late spectral type ( $\mathrm{K}$ and $\mathrm{M}$ ). Firstly we observed each target in a narrow band filter for a few minutes in order to obtain a nonsaturated point spread function (PSF). Secondly, we obtained deep ( $\sim 20$ minutes) $\mathrm{K}_{s}$ and $\mathrm{H}$ broadband observations of each target. Data were reduced using standard procedures to eliminate bad pixels, flat and background sky (IRAF and ECLIPSE). A dedicated pipeline has been employed to filter the low spatial frequencies in order to improve the detection limit at subarcsec distances (Masciadri et al. (2005)). Two different methods were used for the low spatial frequencies filtering.

We averaged the contrast $\Delta M$ obtained at 0.5 and 1 arcsec for all the observed targets having a total integration time within the range 20-30 minutes. We find, in the $K_{s}$ band, a $\Delta M=9 \mathrm{mag}$ at 0.5 arcesc and a $\Delta M=11.5 \mathrm{mag}$ at 1 arcsec. We find, in $H$ band, a $\Delta M=9.2 \mathrm{mag}$ at $0.5 \operatorname{arcsec}$ and a $\Delta M=11.7 \mathrm{mag}$ at 1 arcsec. We recall that 0.5 arcsec corresponds, for our sample, to projected separations of 2-35 AU, 1 arcsec to projected separations of 4-70 AU. This illustrates nicely that NACO is a well performing instrument for high-contrast imaging observations.

No very promising massive-planet candidate was identified in our survey. Besides this we calculated the detection limit for 28 of these targets (Masciadri et al. 2005) and we retrieve the upper limit of the star/planet projected separation calculated for a potential 10 and $5 \mathrm{M}_{J}$ planet (Masciadri et al. 2005, Table 4). By analysing these results from a statistical point of view, we can calculate (Masciadri et al. 2005, Fig.9) the cumulative distribution of the upper limit of the distance star/exo-planet for our sample of targets. The thin line represents the cumulative distribution obtained for $5 \mathrm{M}_{J}$ planets. We find that, with respect to our sample of targets, in $50 \%$ of cases (median value), there are not planets at distances larger than $14 \mathrm{AU}$ and, in $100 \%$ of cases, there are no planets at distances larger than $65 \mathrm{AU}$. We underline that we are considering a $5 \mathrm{M}_{J}$ planet just as a reference, but the same calculation could be done for more and/or less massive planets 
in the range 3-10 $\mathrm{M}_{J}$. In the case of more massive planets, the cumulative distribution should have a medium value $(50 \%)$ associated to an angular separation smaller than $14 \mathrm{AU}$. In the case of less massive planets, the cumulative distribution should have a medium value $(50 \%)$ associated to a angular separation larger than 14 AU. To better appreciate the sensitivity with respect to the mass of the planets we also calculated the cumulative distribution for $10 \mathrm{M}_{J}$ planets (Fig.4, bold style line). We find that, in $50 \%$ of the cases (median value) there are no planets at distances larger than $8.5 \mathrm{AU}$ and in $100 \%$ of the cases there are not planets at distances larger than $36 \mathrm{AU}$. The number given in Table 4 (Masciadri et al. 2005) and the cumulative distribution illustrate that it is relatively unlikely that a massive planet exist beyond a certain distance from the star. For example the fact that no $5 \mathrm{M}_{J}$ planet was found for 18 of our targets beyond $20 \mathrm{AU}$ (see Table 4, Masciadri et al. 2005) means that the likelood for the existence of such planets beyond this distance is $\sim(1 / 18) \%$ i.e. $\sim 5.6 \%$. This last estimation is useful for the reader to have an idea of how much the size of the sample affects the statistical results. We remind that we are using the term probability in a less conventional way. A precise (statistically speaking) definition of probability should need some positive detection that we obviously do not have.

\section{Contrast at sub-arcsec distances: comparison of different techniques}

In the survey carried out with NACO (Masciadri et al. 2005) no interesting candidates were detected but typical contrasts at 0.5 arcsec and 1 arcsec were calculated using the detection limit of 28 targets in $\mathrm{H}$ and $\mathrm{K}_{s}$ bands (see previous Section). The success of the search for extrasolar planets strictly depends on how efficient we are in increasing the reachable contrast at sub-arcsecond distances. We calculated, therefore, for deep direct images, the contrast vs. angular separation for different exposure times. Figure 1 (left) shows the result of this calculation. At distances larger than 1 arcsec, the larger is the exposure time, the larger is the reachable contrast. At sub-arcsec distances, the increasing of the exposure time does not supply a better contrast because we are fundamentally limited by the speckle noise. The Simultaneous Differential Technique (SDI) proposed by Racine et al. (1999) is a rather efficient method to reduce the speckle noise at small angular separations from the central star. Images taken simultaneously at slightly different wavelengths are subtracted from each other. Since the speckle noise of both images is nearly the same $\dagger$, the speckle noise is eliminated in the resulting image providing one can sufficiently limit static aberrations. The differential technique finds a useful application in detecting cool methane-rich faint objects. Rosenthal et al. (1996) first proposed observations of the same star done simultaneously in two different wavelengths which are located "on" and "off" the methane $\left(\mathrm{CH}_{4}\right)$ absorption band. If one subtracts the image taken in the absorption band from the one taken outside the absorption band, planet/brown dwarf features appear in the continuum. The absorption at $1.62 \mu \mathrm{m}$, in the $\mathrm{CH}_{4}$ band, is a clear signature of any object having temperature $\mathrm{T} \leqslant 1300^{\circ} \mathrm{K}$ (giant exo-planets and T-type brown dwarfs). Supported by the preliminary results obtained by Close et al. (2004) with the commissioning data of the SDI/NACO instrument we decided to start a new survey in collaboration with the Close group (see Biller contribution, this Proceedings). In this contribution we intend to study the efficiency of the different observational strategies at sub-arcseconds distances from the target to estimate the improvement obtained with the

$\dagger$ We precise that speckles of images taken at slightly different wavelengths match if frames are rescaled opportunely by a $\lambda_{2} / \lambda_{1}$ factor. 
Table 1. Mean contrast at 0.5 arcsec calculated with different observational techniques (deep direct imaging and SDI). ${ }^{(a)}$ We remind to the reader that the SDI technique is employed in narrow band.

\begin{tabular}{ccccccc}
\hline $\begin{array}{c}\mathrm{D} \\
(\mathrm{m})\end{array}$ & Obs. Tech. & Filter & $\begin{array}{c}\Delta m \\
(\mathrm{mag})\end{array}$ & $\mathrm{N}^{\circ} \sigma$ & $\begin{array}{c}\text { Exp. Time } \\
(\text { minutes })\end{array}$ & $\mathrm{N}^{\circ}$ Targets \\
\hline 8 & Deep Imaging NACO $^{(a)}$ & $\mathrm{H}$ & 9.20 & $5 \sigma$ & $20-30$ & 28 \\
8 & SDI/NACO $^{(a)}$ & $1.57 \mu \mathrm{m}$ & 8.61 & $5 \sigma$ & $10-20$ & 15 \\
8 & SDI/NACO & $\mathrm{H}$ & 10.31 & $5 \sigma$ & $10-20$ & 15 \\
\hline
\end{tabular}
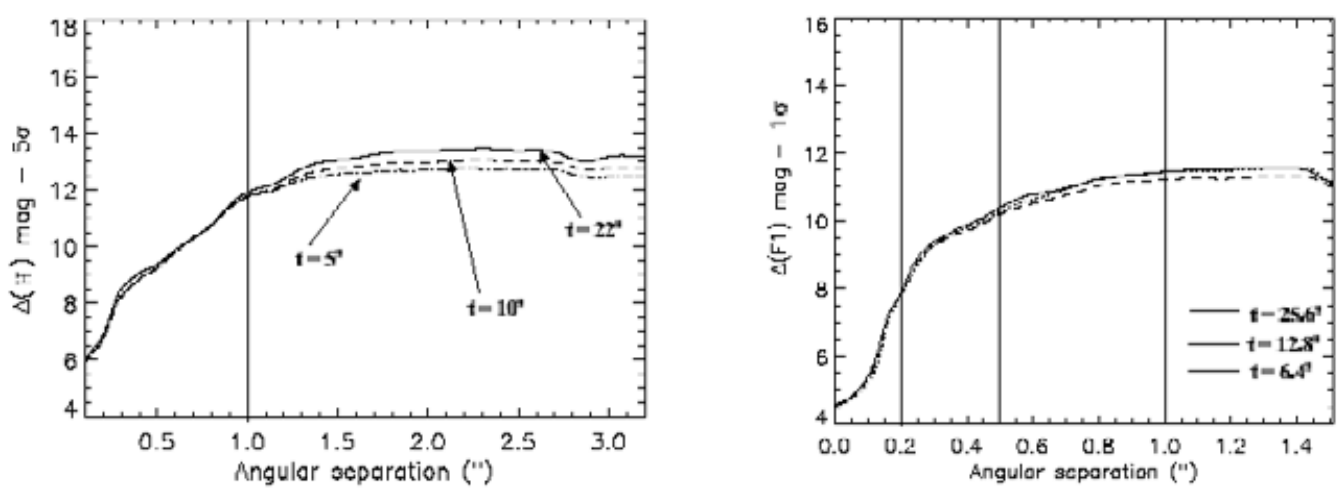

Figure 1. Left: detection limit vs. angular separation of one target of the survey done with deep direct imaging (Masciadri et al. 2005) obtained with integration times of 5, 10 and 22 minutes. Right: detection limit obtained with the SDI technique and a total exposure time of $6.4,12.8$ and 25.6 minutes.

SDI on sky on a $8 \mathrm{~m}$ class telescope. Table 1 summarize the mean contrast calculated at 0.5 arcsec employing the deep direct imaging and the SDI technique running with the same instrument (NACO). We want to precise that, employing the SDI technique, the calculation of the contrast refers to the narrow band, more precisley the narrow band at $1.57 \mu \mathrm{m}(\mathrm{FWHM}=0.024 \mu \mathrm{m})$. In order to compare the two observational strategies (Direct deep imaging and SDI) it is necessary to calculate the equivalent contrast in $\mathrm{H}$ broadband. This means that $\Delta m_{H}=\Delta m_{1.57 \mu m}+\mathrm{A}$. The factor A depends on the spectral type of the planet-like object because the spectrum shape in proximity of the absorption band is different for different spectral type planet-candidates. In the case of a T6 type object we calculated that $\mathrm{A}=1.7 \mathrm{mag}$. For spectral type later than $\mathrm{T} 6, \mathrm{~A}$ is larger than $1.7 \mathrm{mag}$, for spectral type earlier than T6, A is smaller than 1.7 mag.

Looking at Table 1 we conclude that the SDI technique permits to gain a factor of $1 \mathrm{mag}$ in $\mathbf{H}$ band with respect to the Direct deep imaging at 0.5 arcsec. We underline that this gain is a typical value, obtained averaging detection limit curves related to 15 targets and it is valid for the SDI applied to a $8 \mathrm{~m}$ telescope. Figure 1 (right) shows the detection limit at $\mathrm{F}_{1}=1.57 \mu \mathrm{m}$ obtained for different exposure time: $6.4,12.8$ and 25.6 minutes. One can observe that, in the range $0.5-1$ arcsec the gain is $\Delta \mathrm{F}_{1} \sim 0.5 \mathrm{mag}$ in $\Delta \mathrm{t}=15$ minutes. In the range $0.2-0.5 \operatorname{arcsec}$, the gain $\Delta \mathrm{F}_{1}$ is $\sim 0$ mag in the same $\Delta \mathrm{t}$. This means that, at least in the actual version, the gain of the SDI is weakly depending on the total exposure time. 

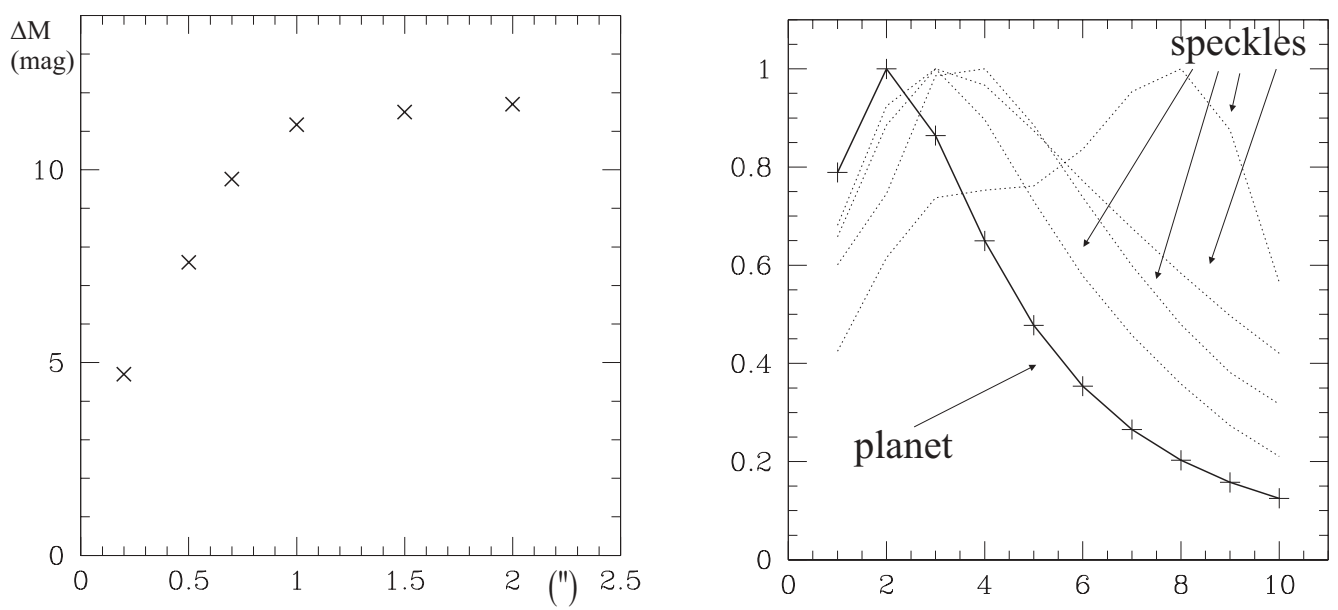

Figure 2. Left: $\Delta H$ (mag) between the central star and the template planet recognized by the automatic procedure vs. the angular separation (arcsec). Right: Example of a normalized spectrum of a planet (y-axis) vs. number of pixels (x-axis). This spectrum is used as template spectrum to recognize planets in a deep image. Dotted lines: spectra of a few speckles recognized by the procedure as local maxima and rejected because they did not follow the criteria of selection.

\section{Automatic recognition of sub-stellar PSFs with wavelet technique}

The fundamental problem for planets detection done with the direct imaging technique is that we have a huge contrast at very small angular separations. It would be therefore quite useful to develop a procedure, better if this is automatic, for recognition of features typical of sub-stellar PSFs. The method that we proposed (Masciadri \& Raga 2005) is based on the wavelet technique. The detailed description of such a procedure can be found in Masciadri \& Raga (2005). The principle is summarized here: a template planet, obtained scaling opportunely the no-saturated PSF of the star, is added in the deep image of the central star. The resulting deep image is filtered from the low spatial frequencies. The procedure characterizes the spectrum of the template planet (Fig.2-right, solid line) and then it looks for, in the deep image, features having the same spectra. These features have to be considered as potential sub-stellar or stellar -like PSF. A second epoch of observation is, therefore, necessary to discriminate between the two cases. This procedure was recently validated (Masciadri \& Raga 2005) on a target (V343 Nor, $\mathrm{m}_{k}=6.3 \mathrm{mag,}$ $d \sim 40 \mathrm{pc}$, age $\sim 12 \mathrm{Myr}$ ) of our NACO survey. Figure 2-right shows the contrast $(\Delta H)$ between the central star and the template planet versus the angular separation within the $0.2-2$ arcsec range at which a planet is recognized by the automatic procedure. We note that, in the case of V343 Nor, a maximum $\Delta H=4.7 \mathrm{mag}$, associated to a planet, can be recognized in an automatic way at 0.2 arcsec with the present procedure. To give an idea to the reader of what we are talking about, we note that 4.7 mag is roughly the contrast in K band of 2M1207, the giant planet candidate recently discovered by Chauvin et al. (2004), with respect to its central star. Figure 2-right shows the spectra of a few "speckles" (dotted lines) recognized by the procedure as local intensity maxima that are rejected by the procedure because their spectra do not follow the criteria of selection (see 'tolerance criterium' in Masciadri \& Raga 2005). The spectrum shown in bold style (Fig.2-left) refers to the template planet placed in the deep image. One can observes that the procedure can discriminate the spectra of planets and speckles in an efficient way. 


\section{Conclusions}

We can summarize the main results obtained in this study in the following way:

- $5 \mathrm{M}_{J}$ planets, having an age within $200 \mathrm{Myr}$, orbiting around stars placed at a distances $d \leqslant 70$ pc can be detected with NACO at distances smaller than 10 AU.

- We find a low probability $(\sim 10 \%)$ to find $5 \mathrm{M}_{J}$ planets at distances larger than 26 $\mathrm{AU}$ and $10 \mathrm{M}_{J}$ at distances larger than $16 \mathrm{AU}$ around stars with properties defined above.

- The performances of different techniques (running at present on sky) at sub-arcsecond distances can be summarized in the following way. At 0.5 arcsec, the SDI technique permits to reach a typical contrast at $5 \sigma$, in [10-25] minutes, equal to $\Delta \mathrm{H}=10.31 \mathrm{mag}$. This is around 1 magnitude better than what obtained with the deep direct imaging $(\Delta H=9.2$ mag). Results published recently (Boccaletti et al. 2004) indicate that the 4 quadrant phase mask (4QPM) coronograph implemented on NACO gives, at 0.5 arcseconds, a $\Delta H=9 \mathrm{mag}$ at $5 \sigma$ in $\mathrm{K}$ band. In order to do a fair comparison with the deep imaging and the SDI technique, results obtained in $\mathrm{H}$ band should be necessary. Considering that the SDI and the 4QPM are certainly techniques that will support the high-contrast future facilities of $8 \mathrm{~m}$ class telescopes and ELTs, we think that it is quite fundamental to verify on sky the performances of these techniques to be able to know where and how to improve such techniques and to scale performances at different parameters space.

At present we can state that the SDI can be considered a success giving access to an improvement of the contrast at sub-arcsecond distances of around 1 mag at 0.5 arcsecond with respect to the deep direct imaging observational strategy. On the other side, we have to note that, no planet candidates have been observed so far with the SDI technique that have not been also observed with the simpler deep direct imaging strategy. From an astrophysical point of view, it would be for sure interesting to be able to do that to optimize the scientific impact of such a promising technique.

- The wavelet technique is certainly a promising method for recognizing, in an automatic way, sub-stellar planet features in deep images.

\section{References}

Baraffe, I., Chabrier, G., Barman, T.S., Allard, F., \& Hauschild, P.H. 2003, A\&A, 402, 701

Boccaletti, A., Riaud, P., Baudoz, P., Baudrand, J., Rouan, D., Gratadour, D., Lacombe, F., \& Lagrange, A.,-M. 2004, PASP, 116, 825

Burrows, A., Marley, M., Hubbard, W.B., Lunine, J.I., Guillot, T., Saumon, D., Freedman, R., Sudarsky, D., \& Sharp, C. 1997 ApJ, 491, 856

Chauvin, G., Lagrange, A.M., Dumas, C., Zuckerman, B., Mouillet, D., Song, I., Beuzit, J.-L. \& Lowrance, P. 2004, A\& 4 AL, 425, L29

Close, L.M., Lenzen, R., Biller, B., Brandner, W., \& Hartung M., 2004, ESO Workshop "Science with $A O ", 136$

Marcy, G.W., Butler, R.P., Fischer, D.A., \& Vogt, S.S. 2003 ASP Conf., 294, 1

Masciadri, E., Mundt, R., Henning, Th., Alvarez, C., \& Barrado y Navascues, D. 2005, ApJ 625,1004

Masciadri, E. \& Raga, A. 2004, ApJL 611, L137

Papaloizou J.C.B. \& Terquem, C. 2001, MNRAS, 325, 221

Racine R., Walker G.A.H., Nadeau D., Doyon R., \& Marois C. 1999, PASP, 111, 587

Rasio, F.A. \& Ford, E.B. 1996, Science, 274, 954

Rosenthal, E.D., Gurwell, M.A., \& Ho, P.T.P 1996, Nature, 384, 243

Weidenschilling, S. J. \& Marzari, F. 1996, Nature, 384, 619 